\title{
La rentabilité et la force économique des bibliothèques publiques québécoises
}

\section{The Profitability and Economic Strength of Quebec's Public}

Libraries

\section{La rentabilidad y la fuerza económica de las bibliotecas públicas quebequenses}

\section{Yvon-André Lacroix}

Volume 39, numéro 4, octobre-décembre 1993

Les bibliothèques publiques

URI : https://id.erudit.org/iderudit/1033311ar

DOI : https://doi.org/10.7202/1033311ar

Aller au sommaire du numéro

\section{Éditeur(s)}

Association pour l'avancement des sciences et des techniques de la documentation (ASTED)

\section{ISSN}

0315-2340 (imprimé)

2291-8949 (numérique)

Découvrir la revue

Citer cet article

Lacroix, Y.-A. (1993). La rentabilité et la force économique des bibliothèques publiques québécoises. Documentation et bibliothèques, 39(4), 215-218. https://doi.org/10.7202/1033311ar
Résumé de l'article

Les bibliothèques publiques québécoises représentent une force économique et elles sont très rentables. Entre autres, elles rapportent plus de 5,4 fois les investissements financiers faits. Par rapport à toutes les autres institutions culturelles québécoises, ce sont les bibliothèques publiques qui coûtent le moins cher aux contribuables et rejoignent le plus l'ensemble de la population québécoise.
Tous droits réservés (c) Association pour l'avancement des sciences et des techniques de la documentation (ASTED),
Ce document est protégé par la loi sur le droit d'auteur. L'utilisation des services d'Érudit (y compris la reproduction) est assujettie à sa politique d'utilisation que vous pouvez consulter en ligne. 


\title{
La rentabilité et la force économique des bibliothèques publiques québécoises
}

\author{
Yvon-André Lacroix* \\ Directeur de la référence \\ Bibliothèque nationale du Québec
}

Les bibliothèques publiques québécoises représentent une force économique et elles sont très rentables. Entre autres, elles rapportent plus de 5,4 fois les investissements financiers faits. Par rapport à toutes les autres institutions culturelles québécoises, ce sont les bibliothèques publiques qui coûtent le moins cher aux contribuables et rejoignent le plus l'ensemble de la population québécoise.

\section{The Profitability and Economic Strength of Québec's Public Libraries}

Québec's public libraries constitute an economic strength and are very profitable. In fact, the return on the investment is about 5,4 times. In comparison with all other cultural institutions in Québec, the public library is the least expensive and is accessible by the most people.

\section{La rentabilidad y la fuerza económica de las bibliotecas públicas quebequenses}

Las bibliotecas públicas quebequenses representan una fuerza económica y son muy rentables. Entre otras cosas, producen más de 5,4 veces de la inversión inicial. En comparación con todas las otras instituciones culturales quebequenses, son las bibliotecas públicas que cuestan menos a los contribuyentes y que sirven más al conjunto de la población quebequense.
Ce texte a fait l'objet d'une conférence dans le cadre du $18 \mathrm{e}$ congrès de l'Asted tenu à Chicoutimi le 28 septembre 1991. Il a été publié dans les Comptes rendus du même congrès sous le titre "Les bibliothèques publiques du Québec: $56^{\circ}$ plus gros employeur au Québec». Les statistiques du présent document concernant les bibliothèques publiques ont été mises à jour.

L'objectif recherché ici est de faire prendre conscience principalement aux responsables des bibliothèques publiques, mais aussi aux élus municipaux et au grand public que, malgré leur importante faiblesse de moyens, les bibliothèques publiques québécoises sont également une richesse économique sur laquelle personne encore, à notre connaissance, ne se soit vraiment attardé. Les bibliothèques publiques sont un levier économique tout autant que les autres institutions culturelles.

Dans les bibliothèques publiques les directeurs sont souvent confrontés à des questions comme celles-ci: La bibliothèque est-elle rentable et comment? Que rapporte la bibliothèqueaux citoyens? Des réponses ont été trouvées et sont exposées ici. Elles proviennent de l'Étude surle financement des arts et de la culture au Québec, publiée en novembre 1990 par la firme Samson, Bélair, Deloitte et Touche.

Le Rapport Samson, Bélair s'étend largement sur l'aspect, entre autres, économique des institutions culturelles québécoises, mais le silence est quasi total sur les bibliothèques publiques. Certes, les bibliothèques publiques québécoises sont très fragiles et se situent encore presque toujours en fin de liste lorsque leurs statistiques sont comparées avec celles des autres bibliothèques publiques des provinces du Canada et des états américains. Malgré cela, et c'est là le paradoxe, elles représentent une force qu'il faut souligner. Et une force économique qui serait plus grande si un bon coup de pouce leur était donné.

Tout comme les musées, les théâtres, les galeries d'art, les bibliothèques publiques sont des institutions économiques.II faut souligner la force et l'apport des bibliothèques publiques à l'éco- nomie: leur efficacité, leur rentabilité. Et rappeler surtout que les bibliothèques publiques sont l'institution culturelle la moins chère comparativement à toutes les autres institutions culturelles.

II n'est donc pas question ici d'élaborer sur la raison d'être fondamentale des bibliothèques publiques et des grands principes de l'Unesco qui sous-tendent leur action. La culture, l'éducation, l'information, la détente pour tous: voilà la grande vocation des bibliothèques publiques.

L'objectif est de cerner une réalité que l'on oublie, que l'on tait, que l'on cache, quel'on ignore. Dans notre société, les bibliothèques publiques sont encore paradoxalement considérées à la fois comme une institution de grande vocation, une institution de luxe et en même temps quelque chose de négligeable, de secondaire, d'accessoire.

\footnotetext{
* Jusqu'au 27 novembre 1992, Y.-A. Lacroix était Directeur du Service de la Bibliothèque municipale de Brossard
} 
Cette vision nous semble quelque peu fausse. Les bibliothèques publiques sont des institutions économiques, sociales, culturelles et politiques vitales pour la société. Les bibliothèques publiques sont fondamentales pour notre société basée sur l'écrit, sur le fait qu'un individu, pour se réaliser, "ne peut pas ne pas lire». Les bibliothèques publiques sont un élément essentiel à la culture en général et à la culture québécoise en particulier.

Cette vision à la fois grandiose et négligeable ne correspond pas à la réalité, en ce sens qu'au niveau quantitatif, les bibliothèques publiques ont des retombées économiques directes immédiates, et au niveau qualitatif, les bibliothèques publiques ont aussi un rendement immédiat.

Rentabilité des investissements au niveau quantitatif

Abordons d'abord l'aspect quantitatif. Donc, chiffres et argent.

Pour le plaisir de la chose, considérons d'abord les bibliothèques publiques comme une entreprise financière. Pour $c e$, consultons l'édition du numéro spécial annuel du journal Les Affaires, publié samedi le 20 juin 1992 intitulé Les 500 plus importantes entreprises au Québec en 1993. Les compagnies y sont inscrites par ordre d'importance du nombre d'employés qu'elles embauchent.

Jetons également un coup d'oeil sur les dernières statistiques de 1991 concernant les bibliothèques publiques $d u$ Québec publiées en 1992. À la page 9, le ministère des Affaires culturelles établit que 2169 employés, équivalent temps plein, travaillent dans les 940 bibliothèques du Québec.

Si nous retournons au journal Les Affaires, édition 1992, cela situe les bibliothèques publiques de cette façon-ci: $48^{e}$ plus gros employeur du Québec (56e en 1991).

De se retrouver en $48^{\mathrm{e}}$ position juste entre les firmes Cascades et Via Rail, ce n'est pas si mal que ça! II ne faut pas penser que ces compagnies soient un accessoire négligeable de notre système économique capitaliste.
En 1991, en effet, ces 2169 employés, équivalent temps plein ${ }^{1}$, gagnent $73663841 \$$ en salaires, soit un salaire moyen de $33912 \$$. Pas mal! Ce serait encore mieux si la norme d'un employé par 2000 habitants était appliquée. Nous créerions alors 1282 autres emplois et la masse salariale serait portée à $117202860 \$$ pour un total de 3450 employés, soit le $27^{\circ}$ plus gros employeur au Québec. Continuons plus loin. Analysons l'impact économique de $2169 \mathrm{em}$ plois. Oublions les 3451 emplois de rêve.

Ainsi, on peut se demander combien d'emplois ces 2169 employés créent-ils? Après enquête, il ne semble pas exister un modèle simplifié et uniforme pour calculer le taux d'emploi généré par un autre emploi. Tout dépend du secteur d'activités. Par exemple, le secteur de la construction effectue plusieurs achats de produits très diversifiés. Un secteur comme le nôtre, les bibliothèques publiques, en est un de service. II génère donc moins d'emplois parce qu'il s'agit ici surtout de salaires.

Néanmoins, on peut affirmer qu'en plus de consommer comme n'importe quel autre consommateur, ces 2169 personnes ont besoin de livres, de périodiques, de cassettes, d'équipements de toutes sortes. Elles font vivre des auteurs, des librairies, des éditeurs, des distributeurs, des agences de périodiques, des relieurs, des camionneurs, des vendeurs de meubles, des papeteries, des compagnies de systèmes informatiques, des concierges, des électriciens, des plombiers, des spécialistes de toutes sortes. Ce personnel a besoin et compte sur les professeurs et les étudiants de l'EBSI et des cégeps offrant les cours de technique en documentation. La liste pourrait s'allonger considérablement sans gros effort.

En termes de mètres carrés occupés par les bibliothèques, le parc immobilier des bibliothèques autonomes représente $194643 \mathrm{~m}^{2}$, soit beaucoup plus que le complexe immobilier de la Place VilleMarie, à Montréal, qui occupe $167220 \mathrm{~m}^{2}$.

Sur le plan des places assises, les bibliothèques publiques autonomes offrent 15506 fauteuils dont 20\% (3 174) pour la seule ville de Montréal. Les trois salles de la Place des Arts de Montréal offrent 5102 fauteuils et les trois salles du
Grand Théâtre de Québec offrent 3000 places assises: soit 8102 places pour ces deux grandes institutions culturelles québécoises. Si ces salles ouvraient 365 jours par année, elles pourraient accueillir au maximum 2957230 personnes.

Dans son Étude sur le financement des arts et de la culture au Québec², publiée en novembre 1990, Samson, Bélair, Deloitte \& Touche estime que les 83 grandes compagnies artistiques québécoises (spectacles) ont accueilli 2663000 spectateurs en 1987 . Or, d'après les dernières statistiques du ministère des Affaires culturelles, 95 des 150 bibliothèques publiques autonomes ont accueilli 7995570 personnes en 1989. Pour la même année, on estime que les 150 bibliothèques ont pu accueillir 12624584 personnes, soit 4,2 fois plus que les salles précitées.

La même étude ${ }^{3}$ établit que les 73 grandes maisons d'édition agréées au Québec ont publié 2700 titres dont 1600 titres scolaires en 1987. Des 1100 titres autres que scolaires, $50 \%$ des titres publiés sont des nouveautés, soit 550 . Ces maisons ont réalisé un chiffre d'affaires de près de 70 millions en 1986-87.

En 1991, les bibliothèques publiques autonomes ont acheté 715355 livres et les BCP 108911 livres, soit un total de 824266 livres pour une valeur de $21817422 \$$, soit $26,47 \$$ le livre $(19,50$ \$ en 1989). Cela représente une moyenne de 4615 livres achetés par les 155 bibliothèques publiques autonomes dans les 217 librairies agréées au Québec, soit $85536 \$$ pour chaque librairie.

Si l'on considère que les bibliothèques publiques consacrent environ $30 \%$ de leur budget à l'achat delivres québécois provenant pour la plupart des 73 grandes maisons d'édition agréées au Québec, il

1. En 1989, 1'Ontario comptait 6248 employés équivalent temps plein dont 1177 bibliothécaires.

2. Étude sur le financement des arts et de la culture au Québec, Montréal, Samson, Bélair, Deloitte \& Touche, novembre 1990.

3. Ibid., p. 286-292. 
Quelques chiffres de 1991 concernant les bibliothèques publiques du Québec

1. Employés

Emplois réels équivalent temps plein

Masse salariale

Salaire moyen

2. Mètres carrés

Bibliothèques publiques

194643

167220

3. Places assises

Bibliothèques publiques

pour accueillir

15506

12624584

6 salles de la Place des Arts et

du Grand Théâtre de Québec

pour accueillir au maximum

8102

2957230

4. Achat de livres

Nombre

Moyenne des achats

par bibliothèque

Moyenne des achats

à chaque librairie

824266 pour $21817422 \$$

4615 livres à $26,47 \$$

$85536 \$$

s'agit là de 214606 livres, soit 390 exemplaires de chacun des 550 grands titres commerciaux québécois de ces maisons.

\section{Une économie de $721589402 \$$}

Les bibliothèques publiques $d u$ Québec permettent aux contribuables québécois de réaliser une ÉCONOMIE FORT SUBSTANTIELLE estimée à $721589402 \$$ pour 1991 . En effet, si nous donnons la valeur moyenne de $26,47 \$$ par
Place Ville-Marie livre acheté par les bibliothèques publiques québécoises en 1991 et si nous multiplions ce montant par le nombre total de prêts, nous arrivons à une dépense de $854002240 \$$ que les abonnés auraient eu à débourser personnellement pour profiter d'un tel avantage. Si nous soustrayons les dépenses nettes de ce montant, incluant les $23065512 \$$ de subventions, soit $132412837 \$$, les Québécois ont ainsi économisé $721589402 \$$, soit

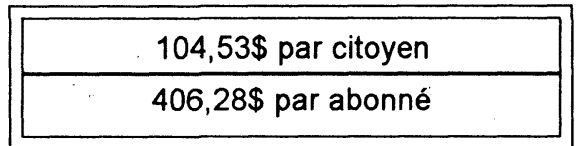

\begin{tabular}{|c|c|c|c|c|}
\hline $\begin{array}{c}\text { Valeur de livres } \\
\text { prétés = nombre } \\
\text { de prêts } x \text { prix du } \\
\text { livre } \\
854002240 \$\end{array}$ & 6903440 & $123,71 \$$ & 1776109 & $480,83 \$$ \\
\hline $\begin{array}{c}\text { Dépenses } \\
\text { totales } \\
133412837 \$\end{array}$ & 6903440 & $19,18 \$$ & 1776109 & $74,55 \$$ \\
\hline $\begin{array}{l}\text { Bénéfices nets } \\
721589402 \$\end{array}$ & 6903440 & $104,53 \$$ & 1776109 & $406,28 \$$ \\
\hline
\end{tabular}

4. Étude sur le financement..., p. 105.

Rentabilité des investissements au niveau qualitatif

Les quelques statistiques sommaires qui précèdent ne correspondent qu'aux documents prêtés à domicile. Elles excluent les millions de documents consultés sur place, les milliers d'actes de référence, les activités d'animation. Elles taisent les coûts évités, la musique écoutée, les vidéos visionnés, etc.

La rentabilité des bibliothèques doit se calculer et s'évaluer tant en quantité qu'en qualité. Les bénéfices monétaires quantifiables sont là. Les bénéfices qualitatifs, ceux qui ajoutent à la qualité de vie, sont également présents. Mais moins évidents. II faut alors lire entre les lignes. Parmi ces bénéfices, impossibles à mesurer scientifiquement et à mettre en chiffres, il y a les bénéfices humains, les connaissances acquises, les inventions... générées par ces connaissances, la culture, l'éducation, la qualité de vie.

Tout cela représente des millions de dollars en retombées économiques, sociales, culturelles, éducatives, intellectuelles et spirituelles... Tout cela grâce à ces milliers et ces milliers de petits services personnels, comptabilisables ou non, rendus par le personnel des bibliothèques publiques du Québec et dont auront profité gratuitement plus de $28,9 \%$ de la population du Québec.

Une brève comparaison: la rentabilité des bibliothèques publiques en regard de celle des autres grands organismes culturels québécois

Jetons un coup d'oeil sur le tableau intitulé "Valeur du billet d'entrée» des grandes institutions culturelles du Québec tiré du Rapport Samson, Bélair". Selon ce tableau, le prix du billet le plus cher varie entre $79 \$$ et $99 \$$ pour un seul spectacle de l'Opéra de Québec. Le prix du billet le moins cher va au Musée de la civilisation, soit $26,00 \$$. Mais la palme pour le billet le moins cher va aux bibliothèques publiques, soit $21,55 \$(18,60$ en 1989$)$ pour autant d'entrées et d'emprunts de livres 
possibles et des dizaines et des dizaines d'heures de lecture.

À ces prix, il faut rajouter, selon le même rapport, certaines autres dépenses. Ainsi, pour une représentation au Théâtre Jean-Duceppe un couple québécois paiera $53,00 \$$ soit $26,50 \$$ le billet. À cela, il faut ajouter $7,00 \$$ pour l'essence et le stationnement; $10,00 \$$ pour la gardienne et souvent $50,00 \$$ pour 2 repas à $25,00 \$$. Une sortie qui coûte $120,00 \$$, soit $60,00 \$$ par personne pour environ 5 heures de culture et de détente.

Pour $86,20 \$$, une famille de 4 personnes pourra se rendre autant de fois qu'elle le voudra à sa bibliothèque publique avec quelques litres d'essence en plus à payer. Qui dit mieux?

Le Rapport Samson, Bélair contient une mine de renseignements que les directeurs des bibliothèques publiques ne devraient pas hésiter à utiliser devant les décideurs et multiplicateurs locaux et na- tionaux. C'est à la lumière de ces chiffres que les conseils municipaux et les élus de l'Assemblée nationale jugeront le passé et décideront de l'avenir et que le ministère des Affaires culturelles n'hésitera peutêtre pas à donner plus que $21,3 \%$ de son budget aux bibliothèques publiques. C'est là une somme résiduelle, nous dit le Rapport Samson, Bélair, malgré leur rendement et les services rendus. Deux grands secteurs accaparent le budget du ministère: les arts d'interprétation (avec $23,4 \%$ ) et les institutions muséales $(28,9 \%)^{5}$.

Faire connaitre ces chiffres et cette réalité a un impact politique direct. C'est se faire connaître, valoir et considérer auprès des autorités, ce qui souvent dans la plupart des cas se traduit par plus de subsides, d'expansion et de visibilité.

\section{Conclusion}

Ces retombées des bibliothèques publiques sont à la fois tellement considérables et subtiles qu'elles deviennent «non calculables». Elles ajoutent à ce qu'on appelle aujourd'hui la qualité de vie. Plus que cela, elles sont à la source de la connaissance. Elles sont la garantie, d'une part, d'une meilleure compétitivité des individus et des institutions dans notre société et, d'autre part, d'une plus grande compétitivité de notre société par rapport à d'autres sociétés. Un relais essentiel à toute forme de participation aux autres activités artistiques, économiques... Ces retombées quantitatives et qualitatives révèlent un monde insoupçonné, mais riche d'une intense activité intellectuelle. Un monde «silencieux», «lowprofile», mais dont personne ne peut se passer. $\mathrm{Ce}$ monde est comme celui d'une marée silencieuse, mais forte, régulière, continue. Un monde intime, mais indispensable au génie humain parce qu'il lui permet de croître. En un mot, c'est le monde fascinant de l'intelligence humaine, des connaissances, du développement et du progrès humain. Le monde d'hier, d'aujourd'hui et de demain.

5. Ibid., p. 79.

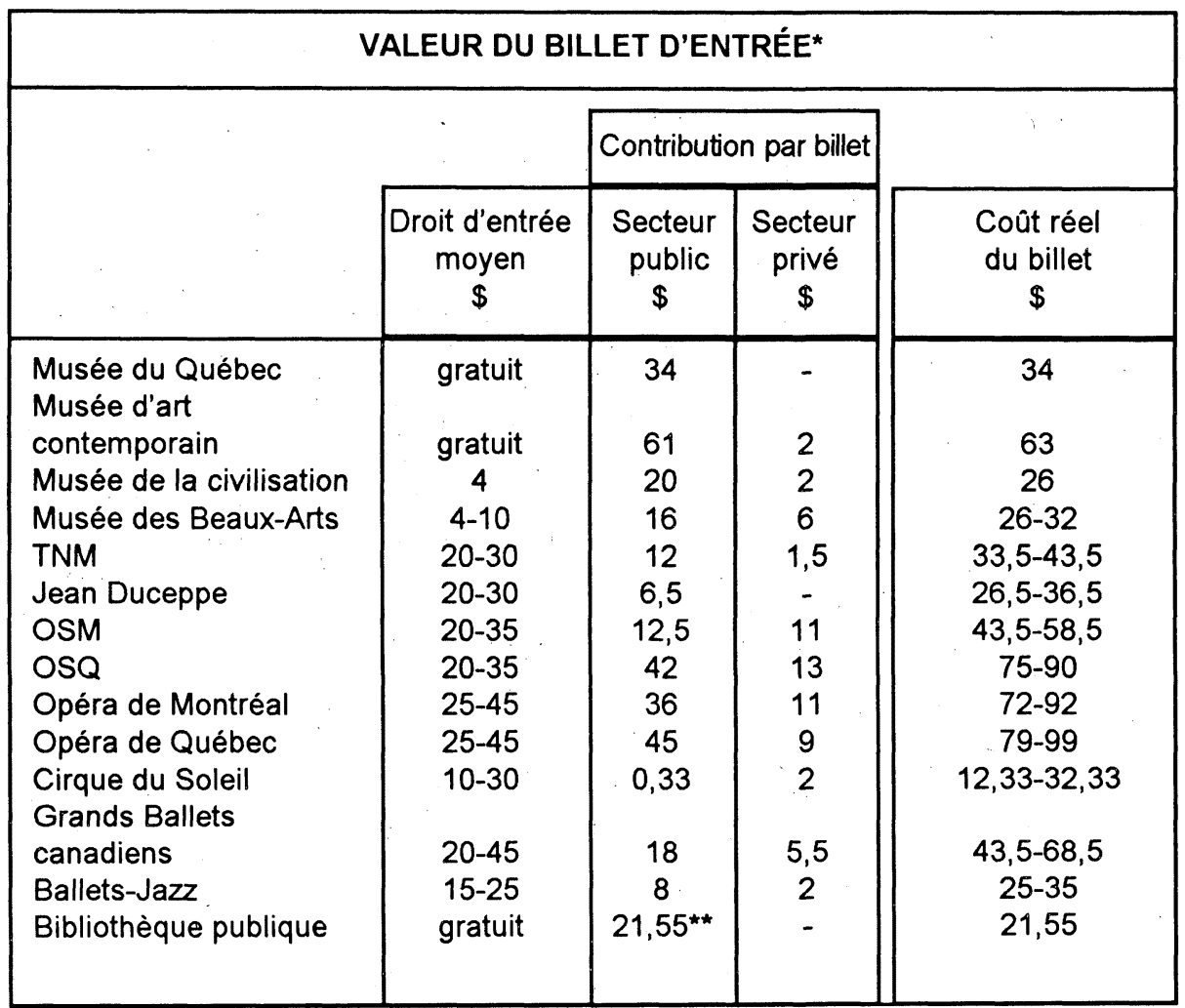

* Sur la base des données de 1988-1989. La dernière colonne de droite a été ajoutée par l'auteur. Extrait du Rapport Samson, Bélair, Deloitte \& Touche

** Chiffres de 1991. Le montant était de 18,60\$ pour 1989. 